\title{
Desenvolvimento de um aplicativo de comunicação de metas entre cuidadores e profissionais de saúde no tratamento de pessoas com Transtorno do Espectro do Autismo
}

\section{RESUMO}

Aline Abreu e Andrade

aline_abreu_andrade@yahoo.com.br Universidade Federal de Minas Gerais, Belo Horizonte, Minas Gerais, Brasil.

Maycoln Leoni Martins Teodoro mlmteodoro@hotmail.com

\begin{abstract}
O tratamento de pessoas com transtorno do espectro do autismo demanda a participação de uma equipe multidisciplinar. Tal equipe conduz intervenções diversas, que devem ser potencializadas no dia a dia da criança, pelos seus cuidadores, o que demanda comunicação de alta frequência dos profissionais entre si e com as famílias. Portanto, o estudo teve como objetivo a construção de uma ferramenta de comunicação e lembrete de metas entre pais e profissionais. Para tal, foi realizada a identificação de fatores facilitadores e dificultadores do uso, a elaboração de uma versão de teste da ferramenta de comunicação e lembrete de metas e um estudo piloto sobre a sua utilização. O estudo teve como resultado a criação do Sistema de Transparência de Equipe entre Pais e Profissionais. Futuras pesquisas deverão ser conduzidas para a investigação da sua aplicação no contexto clínico e da viabilidade de seu uso com diferentes populações.
\end{abstract}

PALAVRAS-CHAVE: Autismo. Comunicação. Metas. Tratamento. Aplicativo. 


\section{INTRODUÇÃO}

As ferramentas de comunicação on-line vêm sendo cada vez mais estudadas como forma de viabilizar interações produtivas e de alta frequência entre equipes de profissionais e o paciente/família. Tais ferramentas podem ser denominadas Tecnologias de Informação e Comunicação em Saúde (TICS). As TICS são definidas pela interação de um indivíduo (consumidor, paciente, cuidador ou profissional) através de um dispositivo de comunicação ou tecnologia eletrônica que permite acessar ou transmitir informações de saúde ou receber orientação e apoio sobre uma questão relacionada à saúde. As TICS podem ter as seguintes funções: transmitir informações, permitir a tomada de decisões embasadas, promover comportamentos de saúde, promover intercâmbio de informações e apoio emocional entre pares, promover o autocuidado e gerenciar a demanda por serviços de saúde. Consequentemente, a característica definidora de uma TICS é que ela não se limita somente ao fornecimento de informações em saúde, mas combina essa informação com pelo menos um serviço adicional (suporte à decisão, apoio comportamental para a mudança ou suporte dos pares) (ENG; GUSTAFSON; HENDERSON; JIMISON, 1999).

Existem muitas abordagens para a avaliação das Tecnologias de Informação e Comunicação em Saúde, todas com o propósito de obter informações sistematizadas para melhorar o design, a implementação, o uso, e a qualidade geral dos programas. A avaliação formativa pode ser usada nos estágios iniciais do desenvolvimento das TICS para avaliar a natureza do problema e as necessidades da população à qual a ferramenta se destina, visando embasar o projeto. Durante as fases de desenvolvimento e implementação, a avaliação de processo pode ser usada para monitorar as características organizacionais, administrativas ou operacionais da intervenção. Já a avaliação dos resultados pode ser usada para examinar a capacidade da ferramenta de alcançar o efeito pretendido em condições ideais ou sob circunstâncias do mundo real e para avaliar sua capacidade de produzir benefícios em relação aos custos. No entanto, é comum que as TICS sejam disponibilizadas ao usuário sem avaliação formal de sua eficácia ou de seu impacto na saúde. Deve-se destacar que ferramentas mal projetadas podem gerar resultados prejudiciais, tais como tratamento inadequado ou atraso na busca de cuidados de saúde. Recomenda-se, assim, uma abordagem baseada em evidências para o desenvolvimento e implementação das TICS (ENG; GUSTAFSON; HENDERSON; JIMISON, 1999).

Então, há demanda pela avaliação de diferentes abordagens para a comunicação on-line entre profissional de saúde e paciente em ambientes clínicos. Tal avaliação deve levar em consideração tanto os resultados do uso de ferramentas sobre os pacientes/famílias quanto sobre os profissionais de saúde. Perguntas adicionais de pesquisa incluem o impacto das ferramentas de comunicação on-line sobre a qualidade dos processos clínicos de cuidados, a adesão às estratégias de intervenção, e a gestão de doenças crônicas (KATZ; MOYER, 2004).

O engajamento da família e dos profissionais é de importância central para a efetividade destas ferramentas, uma vez que é necessária a alimentação frequente de dados na plataforma. Na tentativa de potencializar o uso, mensagens de texto e lembretes para celulares ou e-mail tem sido uma opção cada vez mais popular (NUNDY, 2014). 
Nundy (2014) desenvolveu e implementou durante um período de seis meses um programa chamado CareSmarts. Nele, pacientes com diabetes recebiam mensagens de texto diariamente, visando proporcionar educação sobre a forma de gerir a doença e lembrar os participantes de seguir as medidas de autocuidado recomendadas. Algumas mensagens pediam feedback dos pacientes, de modo a assegurar que eles estavam prestando atenção aos lembretes. O programa apresentou como resultado uma melhora da capacidade de autogestão, levando a um maior controle glicêmico, menos visitas médicas, custos mais baixos e maior satisfação do paciente.

Tradicionalmente, os cuidados em saúde têm sido centrados nas instituições e numa organização top-down (de cima para baixo). Aos poucos, os serviços têm se tornado mais ambulatoriais, e as informações do tratamento, mais compartilhadas. Neste contexto, a definição do trabalho de equipe em saúde como uma rede que liga profissionais e pacientes/famílias em um todo compartilhado parece ser uma descrição mais contemporânea dos modelos emergentes de prestação de cuidados (ROWLEY, 2014).

A comunicação colaborativa é um princípio fundamental que guia familiares e prestadores de cuidados em saúde ao longo do tratamento. Nesta forma de comunicação, objetivos comuns podem ser estabelecidos através de esforços conjuntos. Esse processo permite aos profissionais entenderem mais claramente o contexto atual do tratamento, ajudando a orientar os próximos passos e recomendações de intervenções. Logo ajuda os prestadores de cuidados de saúde e as famílias a alcançarem consenso sobre os rumos do tratamento, o que confere sentido às intervenções (BROWN; CLARK, 2015). Entretanto, apesar da necessidade do estabelecimento destas 'interações produtivas' entre os membros da equipe, tal comunicação pode muitas vezes apresentar problemas, ocorrendo em baixa frequência, com as informações sendo passadas de forma inconsistente entre os profissionais (muitas vezes através da família ou do paciente) e nenhum deles tendo uma compreensão integral do caso (WEINER; BARNET; CHENG; DAALEMAN, 2005).

Dentre os transtornos crônicos que demandam alta frequência de comunicação entre a equipe encontra-se o Transtorno do Espectro do Autismo (TEA), que é caracterizado por déficits persistentes na comunicação e interação social em múltiplos contextos e por padrões restritos e repetitivos de comportamento, interesses ou atividades (APA, 2014). A taxa de prevalência estimada por estudos epidemiológicos atuais para o Transtorno do Espectro do Autismo é de uma pessoa com TEA a cada 64 (CENTER FOR DESEASE CONTROL \& PREVENTION [CDC], 2014). O curso do transtorno é crônico, com prejuízos funcionais ao longo da vida. Em amostras de pessoas com TEA, $80 \%$ dos participantes apresentaram resultados fracos de adaptação social e funcional na vida adulta. Normalmente, os níveis adaptativos parecem estar intimamente relacionados ao suporte familiar, educacional, econômico, comunitário e vocacional (OZONOFF, 2010, ROGERS; OZONOFF; HANSEN, 2013). Também, diante dos avanços no conhecimento sobre os tratamentos, têm sido alcançados resultados cada vez melhores de evolução e adaptação das crianças com TEA (PERRY; COHEN; DECARLO, 1995, ROGERS; OZONOFF; HANSEN, 2013).

A importância do trabalho multidisciplinar no tratamento de pessoas com transtorno do espectro do autismo e suas famílias é enfatizada em muitos planos e diretrizes nacionais, tais como o Plano Nacional do Reino Unido para a Infância 
(NIASA, 2003), as Diretrizes da Nova Zelândia (MINISTRY OF HEALTH AND EDUCATION OF NEW ZEALAND, 2008) e a Linha de Cuidado para Atenção às Pessoas com Transtorno do Espectro do Autismo e suas Famílias na Rede de Atenção Psicossocial do Sistema Único de Saúde (BRASIL, 2013). Conforme destacado anteriormente, um programa de ensino efetivo para uma criança com autismo requer um grupo de profissionais capacitados trabalhando conjuntamente de forma cuidadosa e coordenada, em parceria com os cuidadores, que também são participantes ativos do tratamento (VOLKMAR et al., 2014).

$\mathrm{O}$ trabalho em equipe deve se pautar na presença de metas compartilhadas em relação ao desenvolvimento do paciente, sendo este o objetivo clínico fundamental da comunicação entre os profissionais e a família. A formulação de metas guia a atenção dos indivíduos, seus esforços para determinadas ações, sua persistência e compromisso em fazer o que for necessário para alcançar o objetivo definido (LOCKE; LATHAM, 2002, STAPLES; DILIBERTO, 2010). Estabelecer e alcançar alvos terapêuticos também dá aos envolvidos um senso de propriedade e orgulho sobre seu trabalho. Ademais, a comunicação de metas fornece uma estratégia muito importante para a construção de uma cultura de liderança compartilhada (NEWMAN, 2012).

Gulmans, Vollenbroek-Hutten, van Gemert-Pijnen, e van Gemert-Pijnen (2009) avaliaram a comunicação entre equipe e famílias de crianças com paralisia cerebral, com o objetivo de identificar as lacunas de comunicação relevantes percebidas pelos dois grupos. A ausência de troca de informações foi reconhecida pelos profissionais, que mencionaram certa hesitação em fazer contato com colegas com embasamento teórico ou tipo de abordagem diferente. Os profissionais afirmaram ainda que frequentemente tendiam a pedir aos pais para atuar como mensageiros de informações. Eles reconhecem, no entanto, que alguns pais vivenciam este papel de mensageiro como um fardo, especialmente quando se trata de mensagens orais, que devido a termos técnicos são facilmente mal interpretadas. Já na perspectiva dos pais, os problemas mais frequentemente relatados foram a falta de cooperação e inadequada troca informações centrada no paciente entre os profissionais e a necessidade de atuar como mensageiro de informações entre os profissionais (GULMANS; VOLLENBROEK-HUTTEN; VAN GEMERT-PIJNEN; VAN GEMERT-PIJNEN, 2009). Este último problema destacado é corroborado por outros achados da literatura, em que os pais relataram ser os únicos coordenadores de cuidados para os seus filhos ou a principal forma de comunicação entre os profissionais (MILLER et al., 2009, STILLE; PRIMACK; MCLAUGHLIN; WASSERMAN, 2007).

As lacunas identificadas no estudo de Gulmans, Vollenbroek-Hutten, van Gemert-Pijnen e van Gemert-Pijnen (2009) apontam a necessidade de melhorias focadas na cooperação e troca de informações entre os profissionais, bem como de fatores organizacionais, tais como diretrizes interdisciplinares, definição clara dos papéis e transparência da equipe de tratamento. Uma iniciativa que pode ser promissora a este respeito é a criação de plataformas seguras na internet, onde os pais possam entrar em contato com a rede de profissionais envolvidos no cuidado de seu filho e estes possam interagir entre eles. Na literatura, a ideia de comunicação on-line para melhorar o acesso e transparência dos cuidados em saúde vem ganhando espaço (WEINER et al., 2005). Portanto, o presente estudo teve como objetivo a construção e avaliação inicial da usabilidade do Sistema de 
Transparência de Equipe entre Pais e Profissionais (STEPP), uma ferramenta de comunicação e lembrete de metas entre cuidadores e profissionais.

\section{MÉTODO}

A construção da ferramenta de comunicação entre pais e profissionais foi realizada em três etapas, quais sejam: 1) identificação de fatores facilitadores e dificultadores do uso; 2) elaboração de uma versão de teste da ferramenta e; 3) estudo piloto sobre a sua utilização, visando assegurar a viabilidade do uso do recurso por famílias e profissionais. Com estas três etapas, objetivou-se abranger a avaliação formativa (aferindo as necessidades da população a qual a ferramenta se destina), a avaliação de processo (investigando as características da ferramenta) e a avaliação dos resultados (examinando a capacidade da ferramenta de alcançar o efeito pretendido sob circunstâncias do mundo real) (ENG; GUSTAFSON; HENDERSON; JIMISON, 1999).

\section{IDENTIFICAÇÃO DE FATORES FACILITADORES E DIFICULTADORES DO USO DA FERRAMENTE DE COMUNIDAÇÃO}

A identificação dos fatores facilitadores e dificultadores do uso da ferramenta de comunicação foi feita por meio de três estratégias, descritas a seguir. Inicialmente foi realizada uma revisão da literatura. A busca foi realizada entre os meses de setembro e outubro de 2015, nas seguintes bases: Pubmed, APA PsycArticles, Educational Resources Information Center (ERIC), SpringerLink e SAGE Journals Online. Foram utilizados como termos de busca as palavras: communication, health e team, como parte do título e do resumo. Foram excluídos capítulos de livro e artigos que só possuíam o resumo disponível. Os resumos dos artigos encontrados foram analisados, em busca de pesquisas que abordassem os fatores facilitadores e dificultadores do uso de ferramentas de comunicação entre a equipe e a família no contexto da saúde. Assim, foram selecionados sete artigos, que, ao abordar tais fatores, auxiliaram nas definições sobre a elaboração da ferramenta (GULMANS; VOLLENBROEK-HUTTEN; VAN GEMERT-PIJNEN; VAN GEMERT-PIJNEN, 2009, LOCKE; LATHAM, 2002, NEWMAN, 2012, NUNDY, 2014, ROWLEY, 2014, STAPLES; DILIBERTO, 2010, WEINER et al., 2005).

Como segunda forma de identificação dos fatores facilitadores e dificultadores, houve a condução de um grupo focal com profissionais de saúde. Participaram do grupo quinze especialistas em autismo de diferentes áreas de atuação (um psiquiatra, um terapeuta ocupacional, três fonoaudiólogos e dez psicólogos). Foi feita uma breve introdução ao tema e solicitado que os profissionais discutissem sobre os possíveis fatores facilitadores e dificultadores do uso da ferramenta de comunicação, visando o delineamento de uma primeira versão da plataforma. O tempo de duração do grupo de discussão foi de uma hora. Durante este tempo, foi realizado o registro das variáveis destacadas pelos especialistas.

Finalmente, a terceira estratégia utilizada foi a realização de entrevista com dois familiares de crianças com autismo, sendo um pai e uma mãe. As entrevistas foram realizadas individualmente e tiveram a duração de 30 minutos cada. Nelas, 
foi solicitado que os entrevistados apresentassem a sua opinião sobre os possíveis fatores facilitadores e dificultadores do uso de uma ferramenta de comunicação.

\section{ELABORAÇÃO DE UMA VERSÃO DE TESTE DA FERRAMENTA DE COMUNICAÇÃO E MENBRETE DE METAS}

A ferramenta foi elaborada por uma psicóloga, juntamente com engenheiros de controle e automação. $O$ detalhamento das especificações para a elaboração foi explicitado para todos os envolvidos no processo de construção, visando uma maior compreensão sobre os objetivos da ferramenta de comunicação, bem como dos recursos necessários para o seu funcionamento.

\section{ESTUDO PILOTO DA UTILIZAÇÃO DA FERRAMENTA DE COMUNICAÇÃO E LEMBRETE DE METAS}

Após a elaboração da primeira versão da ferramenta, foi realizado um estudo piloto com três profissionais especialistas de diferentes áreas e dois cuidadores de uma mesma família. Dentre eles, havia uma psicóloga, uma fonoaudióloga, uma terapeuta ocupacional, além da mãe e do pai da criança. Antes de iniciarem sua participação na pesquisa, todos assinaram o termo de consentimento livre e esclarecido e receberam um material explicativo por escrito sobre o tema "comunicação", que foi lido com cada participante. O texto teve como objetivo explicitar aos profissionais e à equipe a importância da comunicação e da adequada elaboração de metas.

Os cinco juízes (três profissionais especialistas de diferentes áreas e dois familiares) utilizaram a ferramenta por duas semanas, visando os ajustes necessários para viabilizar uma alta frequência de uso. Terminado este período, os juízes preencheram um questionário de avaliação sobre a ferramenta. $O$ questionário era composto de imagens de todas as telas e o participante era requisitado a avaliar cada tela individualmente, quanto à qualidade do layout e do conteúdo. O participante tinha as seguintes opções de resposta: inadequado, pouco adequado, adequado e muito adequado, além da possibilidade de acrescentar comentários por escrito em relação a cada tela. Com base nas respostas ao questionário, foram realizadas as alterações necessárias, resultando na versão final da ferramenta.

\section{IDENTIFICAÇÃO DE FATORES FACILITADORES E DIFICULTADORES DO USO DA FERRAMENTA DE COMUNICAÇÃO}

Com base na literatura, foi possível realizar um mapeamento inicial sobre os fatores facilitadores e dificultadores do uso da ferramenta de comunicação. A análise da literatura aponta que a realização da comunicação por meio de plataformas seguras na internet é uma tendência atual, permitindo maior transparência dos cuidados em saúde, além de maior agilidade no contato (WEINER et al., 2005). Portanto, o trabalho de equipe em saúde deve se constituir como uma rede on-line que liga profissionais e pacientes/famílias em um todo compartilhado não hierárquico (ROWLEY, 2014). Tal estruturação evita que se 
atribua aos pais a responsabilidade pela transmissão das informações (GULMANS; VOLLENBROEK-HUTTEN; VAN GEMERT-PIJNEN; VAN GEMERT-PIJNEN, 2009).

Ainda segundo as pesquisas sobre o tema, a construção de uma cultura de liderança compartilhada nos tratamentos em saúde deve passar também pela definição clara de metas, que permite que a comunicação enfoque os temas centrais, não se perdendo em discussões supérfluas (LOCKE; LATHAM, 2002, STAPLES; DILIBERTO, 2010). Então, a comunicação de metas fornece uma estratégia muito importante para a manutenção de uma liderança compartilhada (NEWMAN, 2012). Por fim, as estratégias apontadas para a manutenção da comunicação, dentro da frequência adequada, remetem à facilidade de acesso e de utilização da ferramenta, bem como à solicitação de feedbacks dos usuários (NUNDY, 2014).

Tais informações da revisão de literatura foram complementadas pelos dados coletadas no grupo focal conduzido com quinze profissionais de saúde de diferentes áreas (psiquiatria, terapia ocupacional, fonoaudiologia e psicologia). 0 relato dos participantes foi agrupado em categorias de temas abordados e sintetizados conforme descrito a seguir.

Os especialistas pontuaram como fatores facilitadores do uso da ferramenta: a) a possibilidade de inserção de conteúdo de formas variadas (vídeo, imagem, áudio ou texto); b) a predefinição clara sobre os temas a serem comunicados por meio da ferramenta, de modo a evitar uma banalização do uso, bem como uso exagerado (com frequência muito alta) que inviabilize a utilização por profissionais que acompanham muitas crianças com TEA e; c) o esclarecimento aos profissionais sobre o que são metas de tratamento, já que nem todos têm o hábito de operacionalizar o plano de tratamento em metas.

No entanto, os profissionais relataram como fatores dificultadores do uso da ferramenta: a) o possível incômodo dos profissionais em se expor; b) a explicitação de divergências em relação à condução do tratamento, possivelmente geradora de conflitos e; c) a comunicação poderia se dar de forma confusa ou pouco frequente, caso não houvesse um profissional que exercesse a função de coordenador em relação ao uso da ferramenta.

No intuito de complementar os dados coletados com a opinião dos cuidadores, foi conduzida entrevista individual com dois familiares de crianças com autismo (um pai e uma mãe). Nesta, foram identificados como fatores facilitadores do uso da ferramenta: a) ser acessível pelo celular e; b) a definição sobre os temas a serem comunicados por meio da ferramenta. Em contraposição, os cuidadores apresentaram como potenciais dificultadores do uso da ferramenta: a) o possível incômodo dos profissionais em se expor; b) a explicitação de divergências em relação à condução do tratamento, possivelmente geradora de conflitos e; c) o risco das pessoas se esquecerem de usar o recurso.

Como descrito, alguns fatores apontados pelos profissionais e pelos cuidadores se repetiram. Tal recorrência foi observada nos seguintes aspectos: a) a importância de uma definição clara sobre os temas a serem comunicados através da ferramenta, b) o risco de que os profissionais não a utilizem, devido ao possível incômodo de se expor e; c) a possibilidade da ferramenta gerar conflitos, em decorrência da explicitação de divergências quanto à condução do tratamento. 
Os fatores identificados foram levados em consideração no processo de elaboração da versão de teste da ferramenta, pretendendo criar as condições indicadas dentre os facilitadores, e visando anular ou atenuar os dificultadores. Portanto, foi definido que a ferramenta tecnológica mais indicada seria um aplicativo de celular, com o tema da comunicação bem definido (no caso, o foco de comunicação é especificamente de metas) e com a possibilidade de inserção de conteúdo por vídeo, imagem, áudio ou texto, à escolha do usuário.

Com relação aos fatores dificultadores, buscou-se reduzir o risco de que os profissionais não utilizem a ferramenta com a frequência esperada (a cada sessão do paciente) mediante às ferramentas "Histórico" e "Desempenho", uma vez que tais ferramentas fornecem um feedback para os pais e para os outros profissionais sobre a execução das metas. Logo, a não postagem de metas pelo profissional de saúde impede a execução das mesmas pelos cuidadores, o que pode aumentar a auto-observação do profissional sobre a necessidade de que ele insira as metas com a frequência esperada. Além disso, há a possibilidade de os profissionais programarem um lembrete no aplicativo, para não se esquecerem do uso.

Quanto à clareza da comunicação, foi incluído um tutorial explicitando o que são metas de tratamento, com o intuito de criar condições para que os profissionais passem a operacionalizar o plano de tratamento em metas. Também, a partir da visualização das postagens do restante da equipe os profissionais podem obter modelo sobre a melhor forma de exposição das metas. Assim, quanto mais claras elas estiverem, mais os cuidadores conseguirão executá-las, além de poder ir modelando gradualmente os profissionais sobre as formas mais efetivas de se comunicar. Com isto, objetivou-se dispensar a equipe da necessidade de um coordenador, uma vez que a criação de uma hierarquia pode ampliar a possibilidade de conflitos, bem como aumentar o incômodo do profissional em se expor.

Por fim, o surgimento de possíveis divergências entre os profissionais na condução do tratamento pode ser entendido como um fator dificultador que não será criado pelo aplicativo, mas sim explicitado pela ferramenta. Isto é, muitas equipes de tratamento possuem profissionais com visões muito diferentes, mas tais divergências não ficam tão claras devido à ausência de comunicação entre eles. Na medida em que estas pessoas passam a se posicionar em relação às metas de desenvolvimento que esperam para a criança, o desacordo entre a equipe ficará mais aparente. Sendo assim, levando-se em conta a importância da confluência de pensamentos na equipe, a explicitação das divergências pode ser considerada, inclusive, uma etapa necessária para um maior alinhamento da equipe quanto a condução do tratamento.

A elaboração da versão preliminar da ferramenta foi conduzida pela autora do projeto, juntamente com engenheiros de controle e automação. A definição do layout e do conteúdo das telas foi realizada de forma conjunta, baseada nos critérios destacados na consulta aos especialistas e no levantamento da literatura.

\section{ELABORAÇÃO DE UMA VERSÃO DE TESTE DA FERRAMENTA DE COMUNICAÇÃO E LEMBRETE DE METAS}


operacional e objetiva de metas de estimulação da criança (por parte dos profissionais) e; 2) checklist para lembrete e confirmação sobre a execução das metas propostas na rotina da criança (para os cuidadores). Então, foram subdivididas duas categorias de usuários: 1) cuidadores: composta por pessoas que passam grande parte do dia com a criança (mãe, pai, avô, avó, babá ou outro) e; 2 ) profissionais: constituída de pessoas de diferentes áreas de saúde envolvidas no tratamento (fonoaudiólogo, psicólogo, psiquiatra, terapeuta ocupacional ou outro). Com base nessas duas categorias de usuários, o aplicativo monta uma rede de comunicação focada na criança em tratamento, gerando a comunicação entre os profissionais que acompanham a criança e seus cuidadores.

A introdução das metas é realizada pela equipe por meio de vídeo, imagem, voz ou texto. Cada profissional deve enfocar a descrição de tarefas de estimulação específicas relacionadas ao seu plano de tratamento para a criança. No processo de familiarização dos profissionais com o STEPP, será apresentado um tutorial elucidando exemplos de metas simples e concretas, que possam ser realizadas nos locais onde a criança passa a maior parte de seu dia.

Por exemplo, o psicólogo insere no aplicativo a meta "Sempre que for mostrar um objeto à criança, mostrá-lo perto do seu rosto". A partir de então, todos os outros profissionais têm acesso a esta tarefa, podendo utilizá-las ao longo das sessões de intervenção. Com isto, o profissional pode também deduzir que esta intervenção almeja a meta mais ampla de aumento da frequência e/ou duração do contato ocular.

Os cuidadores, ao receberem diariamente o lembrete, devem estar atentos ao cotidiano para estimularem esta habilidade em alta frequência, aumentando assim a probabilidade de aquisição e generalização do comportamento enfocado. Ao fim do dia, ao receberem o check-list da execução das tarefas, eles são convidados a avaliar o quanto conseguiram ou não implementar as intervenções propostas. Com isto, objetiva-se aumentar a auto-observação e auto monitoramento das pessoas envolvidas no cotidiano da criança, de modo a aumentar a quantidade, qualidade, especificidade e consistência das intervenções realizadas em ambiente natural.

O STEPP possui também um histórico das metas já propostas, apresentado em uma lista constando a data de inserção e o nome do profissional que inseriu a tarefa. Há a possibilidade de busca com base nos filtros "meta por profissional" e "meta por data". Logo, toda a equipe envolvida pode ter acesso às intervenções propostas para a criança ao longo do tempo.

Os usuários têm, ainda, acesso à porcentagem de metas executadas pelos cuidadores em um dado período. Tal informação é apresentada através de um gráfico de execução, onde o eixo y corresponde à porcentagem e o eixo $x$, aos dias. Por exemplo, em uma semana em que foram passadas quatro metas e a família executou diariamente todas as quatro, o gráfico apresenta uma reta horizontal estável, indicando $100 \%$ de execução durante toda a semana.

Há, também, a possibilidade de obter informações qualitativas sobre o checklist de execução das metas pelos cuidadores, permitindo uma avaliação sobre qual tipo de tarefa os cuidadores não conseguiram colocar em prática. Assim, é possível fazer modificações na condução das orientações, aumentando a efetividade das mesmas. 
Será apresentada a seguir a descrição da primeira versão do STEPP, desenvolvida na etapa II do estudo de elaboração do aplicativo. Inicialmente, o usuário deve realizar o seu cadastro. A seguir é solicitada a escolha de um perfil: cuidador ou profissional. Depois, o participante tem acesso aos termos de uso do aplicativo, que contém informações sobre as regras e condições previstas para o uso.

Quando o novo usuário é um cuidador, são apresentadas as opções de escolha entre "Pai", "Mãe", "Avô", Avó", "Babá", "Auxiliar de turma", "Mediador", "Professor" ou "Outro". Neste momento, o cuidador também cadastra o nome da criança que será alvo da estimulação.

Caso o usuário seja um profissional, é apresentada uma tela com as opções mais frequentes das especialidades envolvidas no tratamento (psicólogo, psiquiatra, terapeuta ocupacional, fonoaudiólogo, neurologista, professor), além da opção "Outro", para profissionais de outras áreas.

Após o cadastro, o cuidador pode convidar profissionais e outros cuidadores para a equipe. Ao convidar um novo profissional ou cuidador para a equipe, será enviado um e-mail para o mesmo. Através deste e-mail, o usuário convidado poderá aceitar a solicitação e será incluído na equipe.

Caso o usuário seja um cuidador, ele irá visualizar a equipe referente à sua criança cadastrada. Sempre que o cuidador abrir o aplicativo, terá acesso à tela inicial "Metas em andamento", na qual verá a lista dos profissionais envolvidos no tratamento de sua criança e poderá visualizar a meta atual de cada tratamento.

Figura 1 - Tela inicial do STEPP

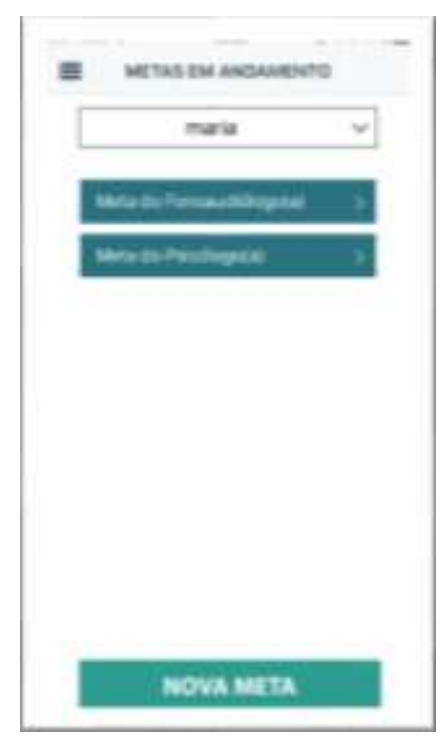

Fonte: Autoria própria.

Ao clicar em Nova meta, o profissional poderá inserir a informação em diferentes formatos (imagem, vídeo, áudio ou texto). Destaca-se que os cuidadores não terão acesso à tela "Nova meta". 
Figura 2 - Tela Nova Meta

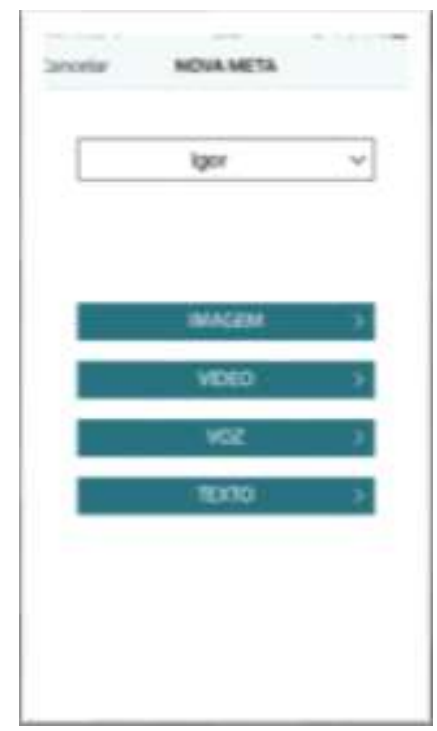

Fonte: Autoria própria.

Ao acessar Metas Concluídas, são exibidas as postagens anteriores. As metas concluídas também são apresentadas mediante uma lista dos profissionais envolvidos no tratamento da criança.

Em relação ao Checklist, diariamente o STEPP envia lembretes para os usuários em forma de uma notificação 'push' (similar à do whatsapp quando uma mensagem é recebida). Uma mensagem é enviada pela manhã lembrando o cuidador de realizar as metas: "não se esqueça das metas do dia". Outra mensagem é enviada no final do dia lembrando o usuário de executar o Checklist: "preencha seu checklist". Portanto, para cada meta em andamento o usuário marca se "fez" ou "não fez". O aplicativo exibe todas as metas, uma após a outra.

Figura 3 - Tela de Checklist

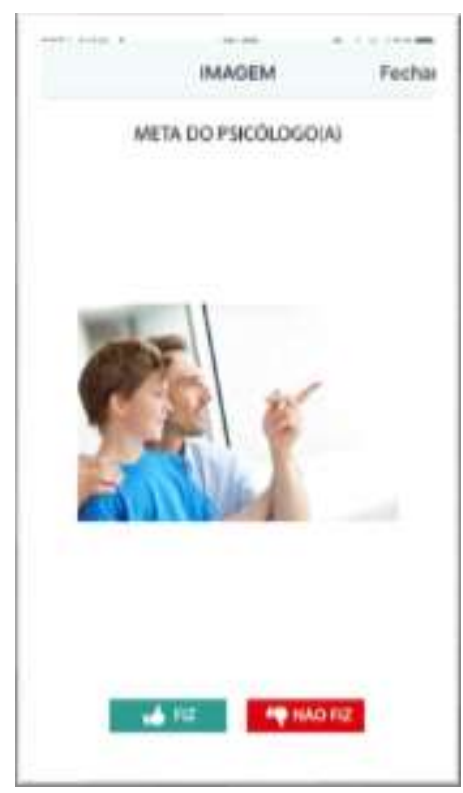


Na última meta apresentada é exibida a informação de que o check-list foi finalizado. Nessa mensagem é exibida uma animação (uma pessoa subindo degraus) para motivar os cuidadores.

\section{ESTUDO PILOTO DA UTILIZAÇÃO DA FERRAMENTA DE COMUNICAÇÃO E LEMBRETE D EMETAS}

No estudo piloto, a primeira versão do aplicativo STEPP foi testada por cinco juízes, que utilizaram a ferramenta por duas semanas. Após este período de uso, os juízes preencheram um questionário de avaliação sobre a ferramenta, no qual foram questionados sobre a qualidade do layout e do conteúdo de cada tela, visando a identificação dos ajustes necessários para viabilizar uma alta frequência de uso da ferramenta.

Os feedbacks apresentados pelos juízes quanto ao grau de adequação das telas foram analisados. Então, pôde-se observar que as alterações sugeridas, referiram-se predominantemente à adequação do layout das telas e acréscimo de conteúdos informativos sobre como utilizar o aplicativo, visando facilitar o seu uso. Todas as ressalvas e sugestões apresentadas são elencadas a seguir.

Em relação à tela de abertura, foi sugerido destacar mais o item "Cadastrar" na tela inicial, de modo que o novo usuário vá direto para este botão, ao invés de preencher o e-mail e a senha (ações adequadas para os usuários já cadastrados). Foi proposto também que o botão "Manter-me conectado" já ficasse marcado na tela inicial, para que o usuário fique automaticamente conectado ao aplicativo ao fazer o cadastro, dispensando uma nova inserção de e-mail e senha, quando do próximo uso do STEPP. Ainda quanto à tela inicial, foi requisitada a inclusão de uma tela de apresentação do aplicativo, visando uma maior compreensão do seu uso.

Quanto à tela de cadastro, houve a sugestão sobre a possibilidade de que os próprios profissionais cadastrassem a criança, ao invés de somente os pais. Foi recomendado também que ao término do cadastro, passasse a aparecer a tela de configuração do horário dos lembretes, explicando a função de cada lembrete e, aumentando a probabilidade do uso desta função do aplicativo.

Através do relato dos juízes, foi possível ainda identificar um erro do STEPP exibido em um dos modelos de celular (Iphone 5S), no qual a opção "Voltar" não era apresentada nas telas "Metas em andamento" e "Check-list". Outra falha identificada foi a marcação errônea no Gráfico de desempenho no check-list, onde, mesmo quando o cuidador marcava que não havia realizado a atividade proposta, o gráfico contava como havendo realizado, ou seja, o preenchimento do check-list, seja com "Fiz" ou "Não fiz", estava sendo contabilizado como acerto.

Todas as críticas e sugestões levaram a alterações no layout e conteúdo das telas. A exceção a isto foi a proposta de que fosse possível que os próprios profissionais cadastrassem a criança, ao invés de somente os pais. Tal proposta não foi acatada por ser incompatível com um dos embasamentos principais do uso do aplicativo, que se centra na ideia de que os pais devem participar ativamente do tratamento. Além disso, a possibilidade de que um profissional realize o cadastro pode colocá-lo no papel de coordenador, trazendo com isto uma hierarquia que pode gerar conflitos (VOLKMAR et al., 2014). 
Ademais, baseado no estudo piloto foi possível estimar que o aplicativo aumenta a frequência de comunicação entre a equipe de tratamento, bem como direciona o tema da comunicação de forma objetiva, gerando o compartilhamento de metas de intervenção. Assim, o STEPP demonstra apresentar o potencial de auxiliar profissionais e cuidadores na condução de um tratamento efetivo.

\section{CONSIDERAÇÕES FINAIS}

O presente estudo teve como resultado a criação do Sistema de Transparência de Equipe entre Pais e Profissionais (STEPP), aplicativo que visa instrumentalizar as equipes de tratamento de pessoas com transtorno do espectro do autismo para o aumento da frequência e qualidade da comunicação em relação às metas de estimulação da criança. $O$ método utilizado no estudo permitiu o mapeamento das necessidades dos futuros usuários, o detalhamento das especificações de recursos necessários, bem como a correção de erros e a condução de melhorias no aplicativo.

Novos trabalhos serão conduzidos, pretendendo o aperfeiçoamento do aplicativo, bem como a investigação sobre os impactos do seu uso na prática clínica. Em relação a isto, espera-se que o aplicativo gere primordialmente um aumento da frequência de comunicação, permitindo uma constância na transmissão de informações relevantes e práticas sobre as intervenções. Esperase, ainda, que tal incremento na comunicação traga consigo um aumento da frequência e qualidade de estimulação da criança nos diversos ambientes. Estas hipóteses serão investigadas em estudos futuros.

Destaca-se que o aplicativo foi concebido inicialmente para uso com equipes de tratamento de pessoas com transtorno do espectro do autismo (TEA), mas pode ter seu uso ampliado para outras populações que requeiram tratamento multidisciplinar e estimulação intensiva. Futuras pesquisas podem abordar também a viabilidade de tal aplicação. 


\title{
Development of a communication goals application to caregivers and health professionals in the treatment of people with Autism Spectrum Disorder
}

\begin{abstract}
The treatment of people with autism spectrum disorder requires the participation of a multidisciplinary team. This team conducts a number of interventions that must be leveraged on the day of the child, by their caregivers, which requires high-frequency communication of professionals among themselves and with their families. Thus, the study aimed to build a communication and reminder goals tool between parents and professionals. For such, it was held to identify facilitating and inhibiting factors of use, the development of a test version of the communication and reminder goals tool and a pilot study on its use. The study resulted in the creation of Team Transparency System between Parents and Professionals. Future research should be conducted for the investigation of their application in the clinical setting and the feasibility of its use with different populations.
\end{abstract}

KEYWORDS: Autism. Comunication. Goals. Treatment. Application. 


\section{REFERÊNCIAS}

AMERICAN PSYCHIATRIC ASSOCIATION. Manual diagnóstico e estatístico de transtornos mentais: DSM-5. (Maria Inês Corrêa Nascimento, M. I. C, et al., Trad.). Porto Alegre: Artmed, 2014.

BRASIL. Linha de Cuidado para Atenção às Pessoas com Transtorno do Espectro do Autismo e suas Famílias na Rede de Atenção Psicossocial do Sistema Único de Saúde. Secretaria de Atenção à Saúde. Departamento de Ações Programáticas Estratégicas. Brasília: Ministério da Saúde, 2013.

BROWN, A.; CLARK, J. D. A parent's journey: Incorporating Principles of Palliative Care into Practice for Children with Chronic Neurological Diseases. Semin Pediatr Neurol, in press, 2015.

CENTER FOR DISEASE CONTROLS AND PREVENTION. Prevalence of autism spectrum disorder among children aged 8 years-autism and developmental disabilities monitoring network, 11 sites, United States, 2012. Morbidity and Mortality weekly report. Surveillance summarie, v. 63, n. 2, p. 1-21, 2014.

ENG, T. R.; GUSTAFSON, D. H.; HENDERSON, J.; JIMISON, H.; PATRICK, K. Introduction to Evaluation of Interactive Health Communication Applications. Am J Prev Med, v. 16, n. 1, p. 10-15, 1999.

GULMANS, J., VOLLENBROEK-HUTTEN, M. M. R., VAN GEMERT-PIJNEN, J. E. W. C., VAN HARTEN, W. H. Evaluating patient care communication in integrated care settings: application of a mixed method approach in cerebral palsy programs. International Journal for Quality in Health Care, v. 21, p. 58-65, 2009.

KATZ, S. J., \& MOYER, C. A. The emerging role of online communication between patients and their providers. J Gen Intern Med, v. 19, p. 978 - 83, 2004.

LOCKE, E. A., \& LATHAM, G. P. Building a practically useful theory of goal setting and task motivation. A 35-year odyssey. Am Psychol, v. 57, p. 705-717, 2002.

MILLER, A. R., CONDIN, C.J., MCKELLIN, W. H., SHAW, N., KLASSEN, A. F., SHEPS, S. Continuity of care for children with complex chronic health conditions: parents' perspectives. BioMed Central Health Services Research, v. 9, p. 242, 2009.

MINISTRY OF HEALTH AND EDUCATION OF NEW ZEALAND. New Zealand Autism Spectrum Disorder Guideline. Wellington: Ministry of Health and Education, 2008. 
NEWMAN, R. Goal setting to achieve results: this school sets goals at four levels-schoolwide, grade level, classroom and individual student--to ensure shared ownership of learning and results. Leadership, v. 41, n. 3, p. 1-12, 2012.

NUNDY, S. Daily text messages and nurse followup improves self- management of diabetes, leading to better glycemic control and lower costs, Agency for Healthcare Research and Quality (AHRQ). Innovations Exchange, 2014. Disponível em: < https://innovations.ahrq.gov/profiles/daily-text-messages-and-nursefollowup-improve-self-management-behaviors-patients-diabetes $>$. Acesso em: 04/2015.

OZONOFF, S. Autism Spectrum Disorders. Em: Yeats, K. O., Ris, M. D., Taylor, H. G. \& Pennington, B. F. Pediatric Neuropsychology: Research, theory and practice. Londres: The Guilford Press, 2010.

PERRY, R., COHEN L., \& DECARLO, R. Case study: deterioration, autism and recovery in two siblings. Journal of the American Academy of Child and Adolescent Psychiatry, v. 34, p. 232-237, 1995.

ROGERS, S. J., OZONOFF, S. \& HANSEN, R. L. Autism spectrum disorders. Em: Hansen, R. L. \& Rogers, S. J. (Eds.) Autism and other neurodevelopmental disorders. American Psychiaty Publishing, 2013.

ROWLEY, R., D. Rowley Professional Social Networking. Curr Psychiatry Rep, v. 16, p. 516-522, 2014.

STAPLES, K. E., \& DILIBERTO, J. A. Working with parents of students with disabilities. Teaching Exceptional Children, v. 42, n. 6, p. 58-63, 2010.

STILLE, C. J., PRIMACK, W. A., MCLAUGHLIN, T. J., \& WASSERMAN, R. C. Parents as information intermediaries between primary care and specialty physicians. Pediatrics, v. 120, p. 1238-46, 2007.

VOLKMAR, F., SIEGEL, M., WOODBURY-SMITH, M., KING, B, MCCRACKEN, J., \& STATE, M. Practice Parameter for the Assessment and Treatment of Children and Adolescents With Autism Spectrum Disorder. Journal Of The American Academy Of Child \& Adolescent Psychiatry, v. 53, p. 2, 2014.

WEINER, S. J., BARNET, B., CHENG, T. L., DAALEMAN, T. P. Processes for effective communication in primary care. Ann Intern Med, v. 142, p. 709-14, 2005. 
Recebido: 20 mar. 2016

Aprovado: 24 out. 2016

DOI: $10.3895 /$ rts.v13n27.3832

Como citar: ANDRADE, A. A. TEODORO, M. L. M. Desenvolvimento de um aplicativo de comunicação de metas entre cuidadores e profissionais de saúde no tratamento de pessoas com Transtorno do Espectro do Autismo. R. Tecnol. Soc., Curitiba, v. 13, n. 27, p. 139-155, jan./abr. 2017. Disponível em:

<https://periodicos.utfpr.edu.br/rts/article/view/3832>. Acesso em: XXX

Correspondência:

Aline Abreu e Andrade.

Rua Aimorés 2710, Santo Agostinho. Belo Horizonte - MG. CEP 30140-072.

Direito autoral: Este artigo está licenciado sob os termos da Licença Creative Commons-Atribuição 4.0 Internacional. 